\title{
New Applications of a Kind of Infinitesimal-Operator Lie Algebra
}

\author{
Honwah Tam, ${ }^{1}$ Yufeng Zhang, ${ }^{2}$ and Xiangzhi Zhang ${ }^{2}$ \\ ${ }^{1}$ Department of Computer Science, Hong Kong Baptist University, Kowloon Tong, Hong Kong \\ ${ }^{2}$ College of Sciences, China University of Mining and Technology, Xuzhou 221116, China
}

Correspondence should be addressed to Yufeng Zhang; zyfxz@cumt.edu.cn

Received 22 January 2016; Accepted 26 June 2016

Academic Editor: Remi Léandre

Copyright (C) 2016 Honwah Tam et al. This is an open access article distributed under the Creative Commons Attribution License, which permits unrestricted use, distribution, and reproduction in any medium, provided the original work is properly cited.

Applying some reduced Lie algebras of Lie symmetry operators of a Lie transformation group, we obtain an invariant of a second-order differential equation which can be generated by a Euler-Lagrange formulism. A corresponding discrete equation approximating it is given as well. Finally, we make use of the Lie algebras to generate some new integrable systems including $(1+1)$ and $(2+1)$ dimensions.

\section{Introduction}

Some research on difference equations admitting Lie-point transformations can be found in the literature $[1,2]$. Specifically, some symmetry-preserving difference schemes for nonlinear differential equations were discovered in [3-5]. We first briefly recall some fundamental notations. Given $L$ satisfying the Euler-Lagrange equation,

$$
\frac{\delta L}{\delta y}=\frac{\partial L}{\partial y}-D\left(\frac{\partial L}{\partial y^{\prime}}\right)=0
$$

where

$$
D=\frac{\partial}{\partial x}+y^{\prime} \frac{\partial}{\partial y}+y^{\prime \prime} \frac{\partial}{\partial y^{\prime}}+\cdots
$$

is a Lie group operator, it is well-known that the functional

$$
\widetilde{L}=\int_{\Omega} L\left(x, y, y^{\prime}\right) d x, \quad \Omega \subset R^{1}
$$

achieves its extremal values on [1]

Equation (1) is an ODE that can be rewritten as

$$
y^{\prime \prime}=f\left(x, y, y^{\prime}\right)
$$

Group $G$ generated by the vector field

$$
X=\xi(x, y) \frac{\partial}{\partial x}+\eta(x, y) \frac{\partial}{\partial y}
$$

is a variational symmetry of the functional $\widetilde{L}$ if and only if the Lagrangian satisfies

$$
\operatorname{pr} X(L)+L D(\xi)=0 .
$$

If there exists a function $V(x, y)$ such that

$$
\operatorname{pr} X(L)+L D(\xi)=D(V),
$$

then the group $G$ is known as an infinitesimal divergence symmetry. By Noether's theorem, one infers that [5]

$$
\begin{aligned}
\operatorname{pr} X(L)+L D(\xi)= & \left(\eta-\xi y^{\prime}\right) \frac{\delta L}{\delta y} \\
& +D\left(\xi L+\left(\eta-\xi y^{\prime}\right) \frac{\partial L}{\partial y^{\prime}}\right) .
\end{aligned}
$$

Therefore, if the vector operator $X$ is a divergence symmetry, we have a first integral

$$
K=\xi L+\left(\eta-\xi y^{\prime}\right) \frac{\partial L}{\partial y^{\prime}}-V
$$

which corresponds to the Euler-Lagrange equation.

In [4], the Lagrangian formalism for second-order difference equations was reviewed. Consider a finite difference functional

$$
\widehat{L}=\sum_{\Omega} \mathscr{L}\left(x, x_{+}, y, y_{+}\right) h_{+}
$$


defined on some one-dimensional lattice $\Omega$ with step spacing $h_{+}$, which can be represented by

$$
h_{+}=\varphi\left(x, y, x_{+}, y_{+}\right) \text {. }
$$

For an arbitrary curve the stationary value of a differential functional is given by any solution of the quasiextremal equations $[4,5]$ :

$$
\begin{aligned}
& \frac{\delta \mathscr{L}}{\delta y}=h_{+} \frac{\partial \mathscr{L}}{\partial y}+h_{-} \frac{\partial \mathscr{L}^{-}}{\partial y}=0, \\
& \frac{\delta \mathscr{L}}{\delta x}=h_{+} \frac{\partial \mathscr{L}}{\partial x}+h_{-} \frac{\partial \mathscr{L}^{-}}{\partial x}+\mathscr{L}^{-}-\mathscr{L} .
\end{aligned}
$$

It can be verified that if the Lagrangian density $\mathscr{L}$ is divergence invariant under group $G$, it holds that

$$
\operatorname{pr} X(\mathscr{L})+\underset{+h}{\mathscr{L} D_{+h}}(\xi)=\underset{+h}{D}(V)
$$

then each element $X$ of the Lie algebra corresponding to $G$ provides us with a first integral of (12):

$$
K=h_{-} \eta \frac{\partial \mathscr{L}^{-}}{\partial y}+h_{-} \xi \frac{\partial \mathscr{L}^{-}}{\partial x}+\xi \mathscr{L}^{-}-V
$$

In the paper, we start from the infinitesimal symmetry operators of an eight-parameter projective transformation group in $R^{2}$ [6],

$$
\begin{aligned}
& X_{1}=x^{2} \frac{\partial}{\partial x}+x y \frac{\partial}{\partial y} \\
& X_{2}=x y \frac{\partial}{\partial x}+y^{2} \frac{\partial}{\partial y} \\
& X_{3}=x \frac{\partial}{\partial x} \\
& X_{4}=y \frac{\partial}{\partial x} \\
& X_{5}=\frac{\partial}{\partial x} \\
& X_{6}=x \frac{\partial}{\partial y} \\
& X_{7}=y \frac{\partial}{\partial y} \\
& X_{8}=\frac{\partial}{\partial y}
\end{aligned}
$$

to discuss some continuous symmetries of Lagrangians and general solutions of discrete equations. We generate new $(1+$ $1)$-dimensional and $(2+1)$-dimensional integrable systems with three potential functions. Specifically, we obtain an integrable coupling of the standard Burgers equation and a $(2+1)$-dimensional integrable coupling of the heat conduct equation. We also derive a $(2+1)$-dimensional integrable coupling of the $(2+1)$-dimensional hyperbolic equation under the framework of the Tu scheme. Finally, we establish a vector Lie algebra to generate expanding integrable models of the $(1+1)$-dimensional and $(2+1)$-dimensional integrable hierarchies of evolution equations obtained in the paper.

\section{A Few Lie Subalgebras of the Operator Algebra}

The Lie algebra (15) has the following commutative operations [6]:

$$
\begin{aligned}
& {\left[X_{1}, X_{2}\right]=0 \text {, }} \\
& {\left[X_{1}, X_{3}\right]=-X_{1} \text {, }} \\
& {\left[X_{1}, X_{4}\right]=-X_{2} \text {, }} \\
& {\left[X_{1}, X_{5}\right]=-2 X_{3}-X_{7} \text {, }} \\
& {\left[X_{1}, X_{6}\right]=\left[X_{1}, X_{7}\right]=0 \text {, }} \\
& {\left[X_{1}, X_{8}\right]=-X_{6} \text {, }} \\
& {\left[X_{2}, X_{3}\right]=\left[X_{2}, X_{4}\right]=0 \text {, }} \\
& {\left[X_{2}, X_{5}\right]=-X_{4} \text {, }} \\
& {\left[X_{2}, X_{6}\right]=-X_{1} \text {, }} \\
& {\left[X_{2}, X_{7}\right]=-X_{2} \text {, }} \\
& {\left[X_{2}, X_{8}\right]=-X_{3}-2 X_{7} \text {, }} \\
& {\left[X_{3}, X_{4}\right]=-X_{4} \text {, }} \\
& {\left[X_{3}, X_{5}\right]=-X_{5} \text {, }} \\
& {\left[X_{3}, X_{6}\right]=X_{6} \text {, }} \\
& {\left[X_{3}, X_{7}\right]=\left[X_{3}, X_{8}\right]=\left[X_{4}, X_{5}\right]=0 \text {, }} \\
& {\left[X_{4}, X_{6}\right]=X_{7}-X_{3} \text {, }} \\
& {\left[X_{4}, X_{7}\right]=-X_{4} \text {, }} \\
& {\left[X_{4}, X_{8}\right]=-X_{5} \text {, }} \\
& {\left[X_{5}, X_{6}\right]=X_{8}} \\
& {\left[X_{5}, X_{7}\right]=\left[X_{5}, X_{8}\right]=0 \text {, }} \\
& {\left[X_{6}, X_{7}\right]=X_{6} \text {, }} \\
& {\left[X_{6}, X_{8}\right]=0 \text {, }} \\
& {\left[X_{7}, X_{8}\right]=-X_{8} \text {. }}
\end{aligned}
$$

From this Lie algebra, some interesting Lie algebras given in [4] can be obtained. In fact, the Lie algebras in [4] denoted by 
$D_{2,1}, D_{2,2}, D_{3,1}, D_{3,2}, D_{3,3}$ are actually linear combinations of the elements in (15):

$$
\begin{aligned}
& D_{2,1}=\left\{X_{5}, X_{8}\right\}, \\
& D_{2,2}=\left\{X_{3}+X_{7}, X_{8}\right\}, \\
& D_{3,1}=\left\{X_{5}, X_{8}, X_{3}+k X_{7}\right\}, \\
& D_{3,2}=\left\{X_{5}, 2 X_{3}+X_{7}, X_{1}\right\}, \\
& D_{3,3}=\left\{X_{5}, X_{8}, X_{3}+X_{6}+X_{7}\right\} .
\end{aligned}
$$

In [4], by using some subalgebras, some difference equations corresponding to Lagrangian invariants and some general solutions of the corresponding quasiextremal equations were obtained. Therefore, it would be important to further study the operator Lie algebra (15) for applications in generating new difference equations and new integrable systems. If we denote the Lie algebra (15) by

$$
g=\operatorname{span}\left\{X_{1}, X_{2}, \ldots, X_{8}\right\}
$$

then it is easy to have

$$
\begin{aligned}
g & =g_{1}+g_{2}, \\
g_{1} & =\operatorname{span}\left\{X_{1}, X_{2}, X_{3}, X_{4}\right\}, \\
g_{2} & =\operatorname{span}\left\{X_{5}, X_{6}, X_{7}, X_{8}\right\},
\end{aligned}
$$

where $g_{1}$ and $g_{2}$ are Lie subalgebras of $g$ and both are not semisimple. In addition, we have

$$
\begin{aligned}
& {\left[g_{1}, g_{1}\right] \subset g_{1},} \\
& {\left[g_{2}, g_{2}\right] \subset g_{2},}
\end{aligned}
$$

$$
\left[g_{1}, g_{2}\right] \text { not in } g_{1}, g_{2} \text {. }
$$

Hence, the Lie algebra $g$ is not a direct sum of the Lie subalgebras $g_{1}$ and $g_{2}$. Because of relation (20), $g$ is not a symmetric Lie algebra. Therefore, $g$ itself cannot be utilized to generate integrable couplings. However, subalgebras of $g$ have the potential and will be investigated in the following. In addition, some new Lie subalgebras can be obtained from (15). For example, we take $X_{3} \in g_{1}$ and $X_{5}, X_{6}, X_{7} \in g_{2}$ to make a linear combination:

$$
\begin{aligned}
\bar{X} & =X_{3}+X_{5}+k X_{6}+X_{7} \\
& =(1+x) \frac{\partial}{\partial x}+(k x+y) \frac{\partial}{\partial y} .
\end{aligned}
$$

It is easy to verify that

$$
g_{5}=\operatorname{span}\left\{X_{5}, X_{6}, X_{7}, X_{8}, \bar{X}\right\}
$$

is a Lie subalgebra of $g$, with the commutative relations

$$
\begin{aligned}
{\left[X_{5}, X_{6}\right] } & =X_{8}, \\
{\left[X_{5}, X_{7}\right] } & =\left[X_{5}, X_{8}\right]=0, \\
{\left[X_{5}, \bar{X}\right] } & =X_{5}+k X_{8}, \\
{\left[X_{6}, X_{8}\right] } & =0 \\
{\left[X_{6}, X_{7}\right] } & =X_{6}, \\
{\left[X_{6}, \bar{X}\right] } & =-X_{8}, \\
{\left[X_{7}, \bar{X}\right] } & =-k X_{6}, \\
{\left[X_{7}, X_{8}\right] } & =-X_{8}, \\
{\left[X_{8}, \bar{X}\right] } & =X_{7} .
\end{aligned}
$$

If we let $k=-1$ and remove the element $X_{5}$ from the Lie algebra $g_{5}$, we get a 4 -dimensional Lie subalgebra

$$
g_{4}=\operatorname{span}\left\{X_{6}, X_{7}, X_{8}, \bar{X}\right\} .
$$

The corresponding commutative operations are given by

$$
\begin{aligned}
& {\left[X_{6}, X_{7}\right]=X_{6},} \\
& {\left[X_{6}, X_{8}\right]=0,} \\
& {\left[X_{6}, \bar{X}\right]=-X_{8},} \\
& {\left[X_{7}, X_{8}\right]=-X_{8},} \\
& {\left[X_{7}, \bar{X}\right]=X_{6},} \\
& {\left[X_{8}, \bar{X}\right]=X_{7} .}
\end{aligned}
$$

In what follows, we shall make use of the Lie subalgebras (22) and (24) to generate second-order differential equations and difference equations that preserve Lie-point symmetries. We derive the general solutions of the corresponding quasiextremal equations. Furthermore, we employ the Lie subalgebras to generate some new $(1+1)$-dimensional and $(2+1)$-dimensional integrable hierarchies of evolution equations.

\section{General Solutions of Some Difference Equations}

A direct calculation yields a second-order ODE corresponding to the vector field $\bar{X}$ on the space $\left(x, y, y^{\prime}, y^{\prime \prime}\right)$ :

$$
y^{\prime \prime}=\frac{1}{1-2 k}\left[k+(1-k) y^{\prime}\right]^{(1-2 k) /(1-k)} \text {. }
$$

This equation can be obtained from the Lagrangian

$$
L=y+\frac{1-2 k}{k}\left[k+(1-k) y^{\prime}\right]^{1 /(1-k)} .
$$


Obviously, the Lagrangian admits the symmetries $X_{5}$ and $X_{8}$ :

$$
\begin{aligned}
& \operatorname{pr} X_{5}(L)+L D\left(\xi_{5}\right)=0, \\
& \operatorname{pr} X_{8}(L)+L D\left(\xi_{8}\right)=1=D(x) .
\end{aligned}
$$

With the help of Noether's theorem we obtain the following first integrals:

$$
\begin{aligned}
J_{1}= & y+\frac{1-2 k}{k}\left[k+(1-k) y^{\prime}\right]^{1 /(1-k)} \\
& -\frac{1-2 k}{k} y^{\prime}\left[k+(1-k) y^{\prime}\right]^{k /(1-k)} \equiv A_{0}, \\
J_{2}= & \frac{1-2 k}{k}\left[k+(1-k) y^{\prime}\right]^{k /(1-k)}-x \equiv B_{0} .
\end{aligned}
$$

Solving the second equation for $y^{\prime}$ in (29) and substituting it into the first equation, we obtain

$$
\begin{aligned}
y(x)= & A_{0}+\frac{2 k-1}{k}\left[\frac{k}{1-2 k}\left(x+B_{0}\right)\right]^{1 / k} \\
& +\frac{2 k-1}{k(1-k)}\left[\frac{k}{1-k}\left(x+B_{0}\right)\right]^{((1-k) / k)^{2}} \\
& \cdot\left[\left(\frac{k}{1-2 k}\left(x+B_{0}\right)\right)^{(1-k) / k}-k\right],
\end{aligned}
$$

which is the general solution to (26).

Let us take the difference Lagrangians

$$
\mathscr{L}=\frac{y+y_{+}}{2}+\frac{1-2 k}{k}[k+(1-k) \underset{+h}{D}(y)]^{1 /(1-k)},
$$

which satisfies

$$
\begin{aligned}
& \operatorname{pr} X_{5}(\mathscr{L})+\mathscr{L} D\left(\xi_{5}\right)=0, \\
& \operatorname{pr} X_{8}(\mathscr{L})+\mathscr{L} D\left(\xi_{8}\right)=\underset{+h}{D}(x),
\end{aligned}
$$

where

$$
\underset{+h}{D}(y) \equiv y_{x}=\frac{1}{h_{+}}\left(\underset{+h}{S-1)}(y)=\frac{y_{+}-y}{x_{+}-x}=\frac{y_{+}-y}{h_{+}} .\right.
$$

Then the variations of $\mathscr{L}$ yield the quasiextremal equations

$$
\begin{aligned}
& \frac{\delta \mathscr{L}}{\delta y}: \frac{h_{+}+h_{-}}{2}+\frac{2 k-1}{k}\left[k+(1-k) y_{x}\right]^{k /(1-k)} \\
& \quad+\frac{1-2 k}{k}\left[k+(1-k) y_{\bar{x}}\right]^{k /(1-k)}=0, \\
& \frac{\delta \mathscr{L}}{\delta x}: \frac{y_{+}-y_{-}}{2}+\frac{1-2 k}{k} y_{x}\left[k+(1-k) y_{x}\right]^{k /(1-k)} \\
& \quad+\frac{2 k-1}{k} y_{\bar{x}}\left[k+(1-k) y_{\bar{x}}\right]^{k /(1-k)} \\
& \quad+\frac{1-2 k}{k}\left[k+(1-k) y_{\bar{x}}\right]^{1 /(1-k)} \\
& \quad+\frac{2 k-1}{k}\left[k+(1-k) y_{x}\right]^{1 /(1-k)}=0,
\end{aligned}
$$

where

$$
\underset{-h}{D}(y) \equiv y_{\bar{x}}=-\frac{1}{h_{-}}(\underset{-h}{S-1})(y)=\frac{y-y_{-}}{x-x_{-}}=\frac{y-y_{-}}{h_{-}}
$$

and $h_{+}, h_{-}$are spatial spacing steps. The case $h_{+}=h_{-}$implies that the grid mesh is uniform. By using formula (14), the first integrals are obtained:

$$
\begin{aligned}
I_{1}= & \frac{2 k-1}{k} y_{x}\left[k+(1-k) y_{x}\right]^{k /(1-k)}+\frac{y+y_{+}}{2} \\
& +\frac{1-2 k}{k}\left[k+(1-k) y_{x}\right]^{1 /(1-k)} \equiv A, \\
I_{2}= & x_{+}+\frac{1-2 k}{k}\left[k+(1-k) y_{x}\right]^{k /(1-k)} \equiv B, \\
I_{3}= & \frac{x_{+}^{2}}{2}+\frac{1-2 k}{k} x+\left[k+(1-k) y_{x}\right]^{k /(1-k)} \\
& -\frac{(1-2 k)^{2}}{k(1-k)}[k+(1-k)] y_{x} \equiv C .
\end{aligned}
$$

Solving $y_{x}$ from the second equation in (37) and then substituting the solution into the third one yields the lattice equation:

$$
\begin{aligned}
& B x_{+}-\frac{1}{2} x_{+}^{2}-\frac{(1-2 k)^{2}}{k(1-k)}\left[\frac{k}{1-2 k}\left(B-x_{+}\right)\right]^{(1-k) / k} \\
& \quad \equiv C .
\end{aligned}
$$

Inserting $y_{x}$ into the first equation in (37) gives rise to the general solution to the quasiextremal equation (35):

$$
\begin{aligned}
y_{+} & =y+\frac{2 k^{k-1} A^{k}}{(1-k)(1-2 k)^{k-1}}[k \\
& \left.-\left(\frac{k}{1-2 k}\left(B-x_{+}\right)\right)^{(1-k) / k}\right],
\end{aligned}
$$

which is defined on the lattice determined by the lattice equation (38).

We now consider the invariant model for (26). It is easy to see that (26) admits a Lie transformation group that can be represented by the Lie algebra $g_{4}$. The group consists of infinitesimal vector fields. The corresponding prolongation operators are given by

$$
\begin{aligned}
\widehat{X}_{6}= & \operatorname{pr} X_{6} \\
= & x \frac{\partial}{\partial y}+x^{+} \frac{\partial}{\partial y^{+}}+h_{+} \underset{+h}{D}(x) \frac{\partial}{\partial h_{+}} \\
& +h_{-} D_{-h}(x) \frac{\partial}{\partial h_{-}},
\end{aligned}
$$




$$
\begin{aligned}
& \widehat{X}_{7}=y \frac{\partial}{\partial y}+y^{+} \frac{\partial}{\partial y^{+}}+\left[\underset{+h}{D}(y)-\underset{h+h}{y_{1} D(0)}\right] \frac{\partial}{\partial y_{x}} \\
& +h_{+} D(0) \frac{\partial}{\partial h_{+}}, \\
& \widehat{X}_{8}=\frac{\partial}{\partial y}+\frac{\partial}{\partial y^{+}}, \\
& \operatorname{pr} \bar{X}=x \frac{\partial}{\partial x}+2 y \frac{\partial}{\partial y}+x^{+} \frac{\partial}{\partial x^{+}}+2 y^{+} \frac{\partial}{\partial y^{+}} \\
& +\underset{+h}{h_{+} D}(x) \frac{\partial}{\partial h_{+}}+\left[\underset{+h}{D}(2 y)-\underset{h+h}{y_{1} D}(x)\right] \frac{\partial}{\partial y_{x}} \\
& +\left[\underset{-h}{D}\left(\begin{array}{c}
\zeta_{1} \\
h
\end{array}\right)-\underset{h}{y_{2} D} D(x)\right] \frac{\partial}{\partial y_{x \bar{x}}},
\end{aligned}
$$

where

$$
\begin{aligned}
& x^{+}=\underset{+h}{S}(x), \\
& y^{+}=y\left(x^{+}\right), \\
& \zeta_{1}=\underset{+h}{D}(2 y)-\underset{h^{+h}}{y_{1} D,} \\
& \underset{h}{y_{1}}=\underset{+h}{D}(y), \\
& \underset{h}{y_{2}}=\underset{-h+h}{D} D(y) .
\end{aligned}
$$

There are a few difference invariants of the Lie algebra $g_{4}$ :

$$
\begin{gathered}
\frac{h_{+}}{h_{-}}, \\
\frac{y^{+}}{y}, \\
y_{1} \\
\frac{h}{y_{2}}, \\
\frac{x^{+}}{x}, \\
\frac{h^{+}}{x^{+}}, \\
y_{2} \\
\frac{h}{y^{2}} .
\end{gathered}
$$

By means of the invariants (42), we can write the following explicit discrete scheme for (26):

$$
y_{2}=\frac{1}{1-2 k}[k+(1-k) \underset{+h}{D}(y)]^{(1-2 k) /(1-k)} .
$$

This scheme is certainly not unique and one can construct another form of the invariant difference equation. In the way presented in [7], we can further investigate some approximate solutions and stabilities by using the von Neumann condition and the Fourier method. This topic is not further discussed in this paper.

\section{New Integrable Dynamical Systems}

In the section we discuss another application of the Lie algebra $g_{4}$ to generate new integrable dynamical systems, including $(1+1)$ and $(2+1)$ dimensions, according to the $\mathrm{Tu}$ and TAH schemes $[8,9]$. We have used this approach before to obtain some integrable systems and the corresponding Hamiltonian structures [10-13]. However, we note that the integrable hierarchies derived in this paper possess three potential functions and are different from those in [913]. The integrable dynamical systems here consist of an integrable coupling of the standard Burgers equation, a $(2+$ 1)-dimensional integrable coupling of the heat conduction equation, and a $(2+1)$-dimensional integrable coupling of a $(2+1)$-dimensional hyperbolic equation.

4.1. $(1+1)$-Dimensional Integrable Systems. We start with a general loop algebra of the Lie algebra $g_{4}$ :

$$
\widetilde{g_{4}}=\operatorname{span}\left\{X_{6}(n), X_{7}(n), X_{8}(n), \bar{X}(n)\right\},
$$

where

$$
\begin{aligned}
X_{i}(n) & =X_{i} \lambda^{n}, \quad i=6,7,8 ; \\
\bar{X}(n) & =\bar{X} \lambda^{n}, \quad n \in \mathbf{Z} .
\end{aligned}
$$

Now we apply $\widetilde{g_{4}}$, introducing the isospectral problem

$$
\begin{aligned}
\varphi_{x} & =U \varphi, \quad U=\bar{X}(1)+q X_{6}(0)+r X_{7}(0)+s X_{8}(0), \\
\varphi_{t} & =V \varphi, \\
V & =\sum_{m \geq 0}\left(b_{m} X_{6}(-m)+c_{m} X_{7}(-m)+d_{m} X_{8}(-m)\right) .
\end{aligned}
$$

The stationary compatibility condition of (46) leads to

$$
\begin{aligned}
& c_{m+1}=-b_{m x}+q c_{m}-r b_{m}, \\
& d_{m+1}=-c_{m x}, \\
& b_{m+1}=d_{m x}+r d_{m}-s c_{m} .
\end{aligned}
$$

Taking three constants $b_{0}=\alpha, c_{0}=\beta$, and $d_{0}=\gamma$, we find that (47) is local. For example, we can get

$$
\begin{gathered}
b_{1}=\gamma r-\beta s, \\
c_{1}=\beta q-\alpha r, \\
d_{1}=0, \\
b_{2}=-\beta q s+\alpha r s, \\
c_{2}=-\gamma r_{x}+\beta s_{x}+\beta q^{2}-\alpha q r-\gamma r^{2}+\beta r s, \\
d_{2}=-\beta q_{x}+\alpha r_{x},
\end{gathered}
$$


Note that

$$
\begin{aligned}
& V_{+}^{(n)}=\sum_{m=0}^{n}\left(b_{m} X_{6}(n-m)+c_{m} X_{7}(n-m)\right. \\
& \left.+d_{m} X_{8}(n-m)\right)=\lambda^{n} V-V_{-}^{(n)} .
\end{aligned}
$$

A direct calculation gives

$$
\begin{aligned}
-\left(V_{+}^{(n)}\right)_{x}+\left[U, V_{+}^{(n)}\right]= & b_{n+1} X_{8}(0)-c_{n+1} X_{6}(0) \\
& -d_{n+1} X_{7}(0)
\end{aligned}
$$

According to the Tu scheme, the zero-curvature equation

$$
U_{t}-\left(V_{+}^{(n)}\right)_{x}+\left[U, V_{+}^{(n)}\right]=0
$$

gives rise to the integrable hierarchy of evolution equations

$$
\left(\begin{array}{l}
q \\
r \\
s
\end{array}\right)_{t_{n}}=\left(\begin{array}{c}
c_{n+1} \\
d_{n+1} \\
-b_{n+1}
\end{array}\right) .
$$

We consider some reduction cases of (52). When we take $n=$ 1 , we have

$$
\begin{aligned}
& q_{t_{1}}=-\gamma r_{x}+\beta s_{x}+\beta q^{2}-\alpha q r-\gamma r^{2}+\beta r s, \\
& r_{t_{1}}=-\beta q_{x}+\alpha r_{x} \\
& s_{t_{1}}=-\beta q s+\alpha r s .
\end{aligned}
$$

Setting $\alpha=\gamma=0$ and $\beta=1$, (53) reduces to

$$
\begin{aligned}
& q_{t}=s_{x}+q^{2}+r s, \\
& r_{t}=-q_{x} \\
& s_{t}=-q s .
\end{aligned}
$$

If we set $\gamma=\beta=0$ and $\alpha=1$, then (53) becomes

$$
\begin{aligned}
& r_{t}=r_{x}, \\
& q_{t}=-q r, \\
& s_{t}=r s,
\end{aligned}
$$

which is an integrable coupling of the convective diffusion equation. Equation (55) is solvable.
When one takes $n=2$, one infers that

$$
\begin{aligned}
q_{t_{2}}= & c_{3} \\
= & \beta(q s)_{x}-\alpha(s r)_{x}-\gamma q r_{x}+\beta q s_{x}+\beta q^{3}-\alpha q^{2} r \\
& -\gamma q r^{2}+\beta q r+\beta q r s-\alpha s r^{2}, \\
r_{t_{2}}= & d_{3} \\
= & \gamma r_{x x}-\beta s_{x x}-2 \beta q q_{x}+\alpha(q r)_{x}+2 \gamma r r_{x} \\
& -\beta(r s)_{x}, \\
s_{t_{2}}= & -b_{3} \\
= & \beta q_{x x}-\alpha r_{x x}+\beta r q_{x}-\alpha r r_{x}-\gamma s r_{x}+\beta s s_{x} \\
& +\beta s q^{2}-\alpha q r s-\gamma s r^{2}+\beta r s^{2} .
\end{aligned}
$$

Setting $\alpha=\gamma=0$ and $\beta=1$, (56) reduces to

$$
\begin{aligned}
& q_{t}=(q s)_{x}+q s_{x}+q^{3}+q r+q r s, \\
& r_{t}=-s_{x x}-2 q q_{x}-(r s)_{x}, \\
& s_{t}=q_{x x}+r q_{x}+s s_{x}+s q^{2}+r s^{2} .
\end{aligned}
$$

If we let $\beta=\gamma=0$ and $\alpha=1$, (56) becomes

$$
\begin{aligned}
& q_{t}=-(r s)_{x}-q^{2} r-r^{2} s, \\
& r_{t}=(q r)_{x}, \\
& s_{t}=-r_{x x}-r r_{x}-q r s .
\end{aligned}
$$

Setting $\alpha=\beta=0$ and $\gamma=1$, (56) gives

$$
\begin{aligned}
& q_{t}=-q r_{x}-q r^{2}, \\
& s_{t}=-s r_{x}-s r^{2}, \\
& r_{t}=r_{x x}+2 r r_{x},
\end{aligned}
$$

which is a new $(1+1)$-dimensional integrable coupling of the standard Burgers equation. It is easy to see that when we take $q=s=0$, (59) reduces to the Burgers equation. We can also single out other integrable systems in addition to (53)-(59), but this is not discussed further.

4.2. $(2+1)$-Dimensional Integrable Systems. The Tu scheme is well-known and no review is considered necessary here. This is not the case for the THA scheme, and we briefly discuss how it is used to generate $(2+1)$-dimensional integrable systems [9]. Let $\mathscr{A}$ be an associative algebra over the field $\mathscr{K}=C$ or $R$, and assume that $\partial: \mathscr{A} \rightarrow \mathscr{A}$ satisfies

$$
\begin{aligned}
\partial(\alpha f+\beta g) & =\alpha \partial f+\beta \partial g, \\
\partial(f g) & =f(\partial g)+(\partial f) g,
\end{aligned}
$$

where $\alpha, \beta \in \mathscr{K}$ and $f, g \in \mathscr{A}$. 
Suppose that $\mathscr{A}[\xi]$ is an associative algebra consisting of the pseudodifferential operators $\sum_{i=-\infty}^{N} a_{i} \xi^{i}$, where $a_{i} \in \mathscr{A}$ and $\xi$ satisfies

$$
\xi f=f \xi+\left(\partial_{y} f\right), \quad f \in \mathscr{A},
$$

from which we have

$$
\begin{aligned}
& \xi^{n} f=\sum_{i \geq 0}\left(\begin{array}{l}
n \\
i
\end{array}\right)\left(\partial^{i} f\right) \xi^{n-i}, \\
& f \xi^{n}=\sum_{i \geq 0}(-1)^{i}\left(\begin{array}{l}
n \\
i
\end{array}\right) \xi^{n-i}\left(\partial^{i} f\right),
\end{aligned}
$$

$n \in \mathbf{Z}$.

Then we take operator matrices,

$$
\begin{aligned}
& U=U(\lambda, \xi, u) \in \mathscr{A}[\xi], \quad u=\left(u_{1}, \ldots, u_{p}\right)^{T}, \\
& V=\sum V_{n} \lambda^{-n},
\end{aligned}
$$

and seek for solutions of $V$ from the stationary zero-curvature equation

$$
V_{x}=[U, V] .
$$

From the expansion

$$
\left\langle V, \frac{\partial U}{\partial u_{i}}\right\rangle=\sum_{i} g_{i}^{(n)} \lambda^{-n}
$$

we obtain a recursion relation among $g^{(n)}=\left(g_{1}^{(n)}, \ldots, g_{p}^{(n)}\right)^{T}$, where

$$
\begin{aligned}
\langle a, b\rangle & =\operatorname{tr} R(a b), \\
R: \mathscr{A}[\xi] & \longrightarrow R^{1}, \\
\sum a_{i} \xi^{i} & \longrightarrow a_{-1} .
\end{aligned}
$$

Finally, we try to find an operator $J$ from the hierarchy

$$
u_{t_{n}}=J g^{(n)},
$$

whose Hamiltonian structure can be generated from the $(2+$ 1)-dimensional trace identity

$$
\frac{\delta}{\delta u_{i}}\left\langle V, U_{\lambda}\right\rangle=\lambda^{-\gamma} \frac{\partial}{\partial \lambda} \lambda^{\gamma}\left\langle V, \frac{\partial U}{\partial u_{i}}\right\rangle, \quad i=1,2, \ldots, p .
$$

Based on the above steps for generating $(2+1)$-dimensional integrable hierarchies of evolution equations, we apply the Lie algebra $g_{4}$ to introduce a Lax pair for matrices $U$ and $V$ :

$$
\begin{aligned}
U & =(\lambda+\xi) \bar{X}(0)+q X_{6}(0)+r X_{7}(0)+s X_{8}(0), \\
V & =b X_{6}(0)+c X_{7}(0)+d X_{8}(0),
\end{aligned}
$$

where

$$
\begin{aligned}
& b=\sum_{m \geq 0} b_{m} \lambda^{-m}, \\
& c=\sum_{m \geq 0} c_{m} \lambda^{-m}, \\
& d=\sum_{m \geq 0} d_{m} \lambda^{-m} .
\end{aligned}
$$

Equation (64) leads to

$$
\begin{aligned}
b_{x} & =-(\xi+\lambda) c+q c-r b, \\
c_{x} & =-(\xi+\lambda) d, \\
d_{x} & =(\xi+\lambda) b-r d+s c .
\end{aligned}
$$

Substituting (70) into (71) yields

$$
\begin{aligned}
& c_{m+1}=-b_{m x}-c_{m} \xi-c_{m y}+q c_{m}-r b_{m}, \\
& d_{m+1}=-c_{m x}-d_{m} \xi-d_{m y}, \\
& b_{m+1}=d_{m x}-b_{m} \xi-b_{m y}+r d_{m}-s c_{m} .
\end{aligned}
$$

Assume that $b_{0}=\alpha \xi^{-1}, c_{0}=\beta \xi^{-1}, d_{0}=\delta \xi^{-1}$, where $\alpha, \beta, \delta$ are constants. We can calculate

$$
\begin{gathered}
b_{1}=-\alpha+(\delta r-\beta s) \xi^{-1}, \\
c_{1}=-\beta+(\beta q-\alpha r) \xi^{-1}, \\
d_{1}=-\delta, \\
b_{2}=\left(-\delta r_{y}+\beta s_{y}-\beta q s+\alpha s r\right) \xi^{-1}+\alpha \xi+2 \beta s-2 \delta r, \\
c_{2}=\left(-\delta r_{x}+\beta s_{x}-\beta q_{y}+\alpha r_{y}\right) \xi^{-1}+\beta \xi-\beta q+\alpha r, \\
d_{2}=\left(\alpha r_{x}-\beta q_{x}\right) \xi^{-1}+\delta \xi,
\end{gathered}
$$

Note that

$$
\begin{aligned}
& V_{+}^{(n)}=\sum_{m=0}^{n}\left(b_{m} X_{6}(n-m)+c_{m} X_{7}(n-m)\right. \\
& \left.\quad+d_{m} X_{8}(n-m)\right)=\lambda^{n} V-V_{-}^{(n)} .
\end{aligned}
$$

Then (64) can be decomposed into

$$
-\left(V_{+}^{(n)}\right)_{x}+\left[U, V_{+}^{(n)}\right]=\left(V_{-}^{(n)}\right)_{x}-\left[U, V_{-}^{(n)}\right] .
$$

The degree of the left-hand side of (75) is $\geq 0$, while the righthand side is $\leq 0$. Therefore, the degrees of both sides are zero. Thus, one infers that

$$
\begin{aligned}
-\left(V_{+}^{(n)}\right)_{x}+\left[U, V_{+}^{(n)}\right]= & R\left(b_{n+1}\right) X_{8}(0) \\
& -R\left(c_{n+1}\right) X_{6}(0) \\
& -R\left(d_{n+1}\right) X_{7}(0) .
\end{aligned}
$$


The zero-curvature equation

$$
U_{t}-\left(V_{+}^{(n)}\right)_{x}+\left[U, V_{+}^{(n)}\right]=0
$$

admits the $(2+1)$-dimensional integrable hierarchy

$$
\left(\begin{array}{l}
q \\
r \\
s
\end{array}\right)_{t_{n}}=\left(\begin{array}{c}
R\left(c_{n+1}\right) \\
R\left(d_{n+1}\right) \\
-R\left(b_{n+1}\right)
\end{array}\right) .
$$

When $n=1$, (78) reduces to

$$
\begin{aligned}
& q_{t}=-\delta r_{x}+\beta s_{x}-\beta q_{y}+\alpha r_{y}, \\
& r_{t}=-\beta q_{x}+\alpha r_{x}, \\
& s_{t}=\delta r_{y}-\beta s_{y}+\beta q s-\alpha s r .
\end{aligned}
$$

When $n=2$, (78) reduces to

$$
\begin{aligned}
q_{t}= & R\left(c_{3}\right) \\
= & 2 \delta r_{x y}+\beta(q s)_{x}-\alpha(s r)_{x}+\beta q_{y y}-\alpha r_{y y}-\delta q r_{x} \\
& +\beta q s_{x}-\beta q q_{y}+\alpha q r_{y}+\delta r r_{y}-\beta r s_{y}+\beta q r s \\
& -\alpha s r^{2}, \\
r_{t}= & \delta r_{x x}-\beta s_{x x}+2 \beta q_{x y}-2 \alpha r_{x y}, \\
s_{t}= & \beta q_{x x}-\alpha r_{x x} \delta r_{y y}+\beta s_{y y}-\beta(q s)_{y}+\alpha(s r)_{y} \\
& +\beta r q_{x}-\alpha r r_{x}-\delta r_{x} s+\beta s s_{x}-\beta s q_{y}+\alpha s r_{y} .
\end{aligned}
$$

When $\alpha=\beta=0$ and $\delta=1,(80)$ reduces to a $(2+1)$ dimensional integrable coupling of the $(1+1)$-dimensional heat conduction equation

$$
\begin{aligned}
& r_{t}=r_{x x}, \\
& q_{t}=2 r_{x y}-q r_{x}+r r_{y}, \\
& s_{t}=-r_{y y}-r_{x} s .
\end{aligned}
$$

When $\beta=\delta=0$ and $\alpha=1$, (80) becomes

$$
\begin{aligned}
& r_{t}=-2 r_{x y}, \\
& q_{t}=-(s r)_{x}-r_{y y}+q r_{y}-s r^{2}, \\
& s_{t}=-r_{x x}+(s r)_{y}-r r_{x}+s r_{y},
\end{aligned}
$$

which is a $(2+1)$-dimensional integrable coupling of the $(2+$ $1)$-dimensional hyperbolic equation.

When $\alpha=\delta=0$ and $\beta=1,(80)$ reduces to

$$
\begin{aligned}
& r_{t}=-s_{x x}+2 q_{x y}, \\
& q_{t}=(q s)_{x}+q_{y y}+q s_{x}-q q_{y}-r s_{y}+q r s, \\
& s_{t}=q_{x x}+s_{y y}-(q s)_{y}+r q_{x}+s s_{x}-s q_{y} .
\end{aligned}
$$

In particular, when we take $r=q=0,(83)$ reduces to

$$
s_{t}=s_{y y}+s s_{x}
$$

which is a $(2+1)$-dimensional Burgers equation, with (83) being its generalized integrable coupling.

\section{Expanding Integrable Models of Integrable Systems (52) and (78)}

In the section we want to enlarge the Lie algebra $g_{4}$ and deduce the expanding integrable models of the $(1+1)$ dimensional integrable hierarchy (52) and the $(2+1)$ dimensional integrable hierarchy (78). Obviously, the Lie algebra $g_{5}$ is the minimum enlarging Lie algebra of $g_{4}$. However, $g_{5}$ cannot generate new integrable dynamical systems based on the isospectral problem (46). That is, by applying the enlarging Lie algebra $g_{5}$ we are not able to obtain new expanding integrable models compared to the Lie algebra $g_{4}$, except for an arbitrary smooth function with respect to $x$. Therefore, we have to enlarge the Lie algebra $g_{5}$ to the following Lie algebra (not unique):

$$
\widehat{g}=\operatorname{span}\left\{X_{3}, X_{4}\right\}+g_{5},
$$

which is also a Lie subalgebra of the large Lie algebra (15). It is easy to verify that the Lie algebra $\widehat{g}$ has the following operation relations:

$$
\begin{aligned}
{\left[X_{3}, X_{4}\right] } & =-X_{4}, \\
{\left[X_{3}, X_{5}\right] } & =-X_{5}, \\
{\left[X_{3}, X_{6}\right] } & =X_{6}, \\
{\left[X_{3}, X_{7}\right] } & =\left[X_{3}, X_{8}\right]=0, \\
{\left[X_{3}, \bar{X}\right] } & =-X_{5}-X_{6}, \\
{\left[X_{4}, X_{5}\right] } & =0 \\
{\left[X_{4}, X_{6}\right] } & =X_{7}-X_{3}, \\
{\left[X_{4}, X_{7}\right] } & =-X_{4}, \\
{\left[X_{4}, X_{8}\right] } & =-X_{5}, \\
{\left[X_{4}, \bar{X}\right] } & =X_{3}-X_{7}, \\
{\left[X_{5}, X_{6}\right] } & =X_{8}, \\
{\left[X_{5}, X_{7}\right] } & =\left[X_{5}, X_{8}\right]=0, \\
{\left[X_{7}, X_{8}\right] } & =-X_{8}, \\
{\left[X_{5}, \bar{X}\right] } & =X_{5}-X_{8}, \\
{\left[X_{6}, X_{7}\right] } & =X_{6}, \\
{\left[X_{8}\right] } & =0 \\
{\left[X_{7}\right] } & =-X_{8}, \\
{\left[X_{6}\right.} & \\
{\left[X_{6}\right.} &
\end{aligned}
$$


In what follows, we first introduce an isomorphism between the Lie algebra $\hat{g}$ and the linear space $R^{7}$. Assume that $a, b \in \widehat{g}$; then $a, b$ can be expressed by

$$
\begin{aligned}
& a=\sum_{i=1}^{6} a_{i} X_{2+i}+a_{7} \bar{X}, \\
& b=\sum_{j=1}^{6} b_{j} X_{2+j}+b_{7} \bar{X} .
\end{aligned}
$$

Suppose that there exists a linear map $f: \widehat{g} \rightarrow R^{7}, \forall a \in$ $\hat{g} \rightarrow\left(a_{1}, \ldots, a_{7}\right)^{T}$. It is easy to see that $f$ is an isomorphism between the linear spaces $\widehat{g}$ and $R^{7}$. Based on the commutative relations of the Lie algebra $\hat{g}$, we define an operation in $R^{7}$ by

$$
\begin{aligned}
& {[a, b]_{R}=\left(a_{4} b_{2}-a_{2} b_{4}+a_{2} b_{7}-a_{7} b_{2}, a_{2} b_{1}-a_{1} b_{2}\right.} \\
& +a_{5} b_{2}-a_{2} b_{5}, a_{3} b_{1}-a_{1} b_{3}+a_{3} b_{7}-a_{7} b_{3}+a_{6} b_{2} \\
& -a_{2} b_{6}+a_{7} b_{1}-a_{1} b_{7}, a_{1} b_{4}-a_{4} b_{1}+a_{4} b_{5}-a_{5} b_{4} \\
& +a_{5} b_{7}-a_{7} b_{5}, a_{2} b_{4}-a_{4} b_{2}+a_{7} b_{2}-a_{2} b_{7}+a_{6} b_{7} \\
& -a_{7} b_{6}+a_{7} b_{1}-a_{1} b_{7}, a_{3} b_{4}-a_{4} b_{3}+a_{7} b_{4}-a_{4} b_{7} \\
& \left.+a_{6} b_{5}-a_{5} b_{6}+a_{7} b_{3}-a_{3} b_{7}, 0\right)^{T} .
\end{aligned}
$$

We can prove that the linear space $R^{7}$ becomes a Lie algebra if it is equipped with the operation (88).

Next, we deduce the expanding integrable models of the integrable hierarchies based on the Lie algebra $R^{7}$.

5.1. Expanding Integrable Model of the Integrable Hierarchy (52). We introduce a loop algebra

$$
\begin{aligned}
\widetilde{R^{7}} & =\left\{\left(x_{1}, \ldots, x_{6}, 0\right)^{T}, x_{i}=\sum x_{i m} \lambda^{m}, x_{i m}\right. \\
& \left.=x_{i m}(t, x), i=1, \ldots, 6\right\} .
\end{aligned}
$$

In terms of $\widetilde{R^{7}}$, we consider the Lax matrices

$$
\begin{aligned}
& U=\left(u_{1}, 0, u_{2}, q, r, s, \lambda\right)^{T}, \\
& V=(0, a, f, b, c, d, 0)^{T},
\end{aligned}
$$

where $\lambda$ is a spectral parameter, $a=\sum_{m \geq 0} a_{m} \lambda^{-m}, b=$ $\sum_{m \geq 0} b_{m} \lambda^{-m}, \ldots$. According to the Tu scheme, a solution to the stationary zero-curvature equation

$$
V_{x}=[U, V]_{R}
$$

for $V$ is given by

$$
\begin{aligned}
c_{m+1} & =-b_{m x}+q c_{m}+\left(u_{1}-r\right) b_{m}, \\
d_{m+1} & =-c_{m x}, \\
b_{m+1}+f_{m+1} & =d_{m x}+q f_{m}+r d_{m}-s c_{m}-u_{2} b_{m}, \\
a_{m+1} & =q a_{m}, \\
f_{m+1} & =-f_{m x}-u_{1} f_{m}+s a_{m}, \\
a_{m x} & =\left(r-u_{1}\right) a_{m} .
\end{aligned}
$$

Taking

$$
\begin{aligned}
& b_{0}=\alpha, \\
& c_{0}=\beta, \\
& d_{0}=\gamma, \\
& a_{0}=\sigma, \\
& f_{0}=\delta,
\end{aligned}
$$

then (92) admits that

$$
\begin{gathered}
a_{1}=\sigma q, \\
b_{1}=\delta q+\gamma r-\beta s-\alpha u_{2}, \\
c_{1}=\beta q+\alpha\left(u_{1}-r\right), \\
d_{1}=0 \\
f_{1}=-\delta u_{1}+\sigma s,
\end{gathered}
$$

Note that

$$
\begin{aligned}
V_{+}^{(n)} & =\sum_{m=0}^{n}\left(0, a_{m}, f_{m}, b_{m}, c_{m}, d_{m}, 0\right)^{T} \lambda^{n-m} \\
& =\lambda^{n} V-V_{-}^{(n)} .
\end{aligned}
$$

A direct calculation yields

$$
\begin{gathered}
-\left(V_{+}^{(n)}\right)_{x}+\left[U, V_{+}^{(n)}\right]_{R}=\left(-a_{n+1}, 0,-f_{n+1},\right. \\
\left.-c_{n+1}, a_{n+1}-d_{n+1}, b_{n+1}+f_{n+1}, 0\right)^{T} .
\end{gathered}
$$

Thus the zero-curvature equation

$$
U_{t_{n}}-\left(V_{+}^{(n)}\right)_{x}+\left[U, V_{+}^{(n)}\right]_{R}=0
$$

has an integrable hierarchy

$$
\left(\begin{array}{c}
q \\
r \\
s \\
u_{1} \\
u_{2}
\end{array}\right)_{t_{n}}=\left(\begin{array}{c}
c_{n+1} \\
d_{n+1}-a_{n+1} \\
-b_{n+1}-f_{n+1} \\
a_{n+1} \\
f_{n+1}
\end{array}\right) .
$$


When we take $u_{1}=u_{2}=0$, (98) reduces to the $(1+1)$-dimensional integrable hierarchy (52). Therefore, (98) is an expanding integrable model of (52). A simple reduction of (98), when $n=1$, has the form

$$
\begin{aligned}
q_{t}= & c_{2} \\
= & -\delta q_{x}-\gamma r_{x}+\beta s_{x}+\alpha u_{2 x}+\beta q^{2}-\alpha q r+2 \delta q \\
& +\frac{1}{q}\left(2 \gamma r q_{x}-2 \beta q_{x} s-2 \alpha q_{x} u_{2}\right), \\
r_{t}= & -\beta q_{x}-\alpha r_{x}-\sigma q^{2}, \\
s_{t}= & \delta q u_{1}-\sigma q s+\beta q s-\alpha s r+\delta q u_{2}+\gamma u_{2} r-\beta s u_{2} \\
& -\alpha u_{2}^{2}, \\
u_{1, t}= & \sigma q^{2}, \\
u_{2, t}= & \delta u_{1 x}+\sigma s_{x}+\delta u_{1}^{2}-\sigma u_{1} s+\sigma q s .
\end{aligned}
$$

5.2. Expanding Integrable Model of the $(2+1)$-Dimensional Integrable Hierarchy (78). We define an associative algebra $R^{7}[\xi]$ consisting of elements $\sum a_{i} \xi^{i}, a_{i} \in R^{7}$. A linear operator on $R^{7}[\xi]$ is given by

$$
\partial: R^{7}[\xi] \longrightarrow R^{7}[\xi]
$$

which satisfies

$$
\begin{array}{r}
\partial(f g)=(\partial f) g+f(\partial g), \\
\partial(\alpha f+\beta g)=\alpha(\partial f)+\beta(\partial g),
\end{array}
$$

where $f, g \in R^{7}[\xi]$ and $\alpha, \beta$ are arbitrary constants. In addition, a residue operator is defined by

$$
\begin{aligned}
\mathscr{R}: R^{7}[\xi] & \longrightarrow R^{1}, \\
\mathscr{R}\left(\sum a_{i} \xi^{i}\right) & =a_{-1} .
\end{aligned}
$$

Based on the above notations, we introduce two Lax matrices on $R^{7}[\xi]$ :

$$
\begin{aligned}
& U=(u, 0, v, q, r, s, \lambda+\xi)^{T}, \\
& V=(0, a, f, b, c, d, 0)^{T},
\end{aligned}
$$

where

$$
\begin{gathered}
a=\sum_{m \geq 0} \lambda^{-m}, \\
b=\sum_{m \geq 0} b_{m} \lambda^{-m},
\end{gathered}
$$

Similar to previous discussions, there exist relations among $a, b, c, d, f$ such that

$$
\begin{aligned}
b_{x} & =-(\lambda+\xi) c+q c+(u-r) b, \\
c_{x} & =-(\lambda+\xi) d+(\lambda+\xi) a-q a, \\
d_{x} & =(\lambda+\xi) b+(\lambda+\xi) f-q f-r d+s c+v b, \\
a_{x} & =(r-u) a, \\
f_{x} & =-(\lambda+\xi) f+s a-u f, \\
0 & =-(\lambda+\xi) a+q d .
\end{aligned}
$$

Substituting (104) into (105) gives rise to

$$
\begin{aligned}
c_{m+1}= & -b_{m x}-c_{m} \xi-c_{m y}+q c_{m}+(u-r) b_{m}, \\
d_{m+1}= & -c_{m x}-d_{m} \xi-d_{m y}, \\
b_{m+1}+f_{m+1}= & d_{m x}-b_{m} \xi-b_{m y}-f_{m} \xi-f_{m y}+q f_{m} \\
& +r d_{m}-s c_{m}-v b_{m}, \\
f_{m+1}= & -f_{m x}-f_{m} \xi-f_{m y}+s a_{m}-u f_{m}, \\
a_{m+1}= & q a_{m}-a_{m} \xi-a_{m y}, \\
a_{m x}= & (r-u) a_{m} .
\end{aligned}
$$

Taking

$$
\begin{aligned}
& b_{0}=\alpha \xi^{-1}, \\
& c_{0}=\beta \xi^{-1}, \\
& d_{0}=\delta \xi^{-1}, \\
& a_{0}=\sigma \xi^{-1}, \\
& f_{0}=\gamma \xi^{-1},
\end{aligned}
$$

we have from (106) that

$$
\begin{aligned}
a_{1} & =-\sigma+\sigma q \xi^{-1}, \\
f_{1} & =\gamma+(\sigma s-\gamma u) \xi^{-1}, \\
d_{1} & =-\delta, \\
c_{1} & =-\beta+\beta q \xi^{-1}, \\
b_{1} & +f_{1}=-\alpha-\gamma+(\gamma q+\delta r-\beta s-\alpha v) \xi^{-1}, \\
b_{1} & =-\alpha-2 \gamma+(\gamma q+\delta r-\beta s-\alpha v-\sigma s+\gamma u) \xi^{-1}, \\
c_{2} & =-\beta q+(\alpha+2 \gamma)(r-u)+\left[-\gamma q_{x}-\delta r_{x}+\beta s_{x}\right. \\
& +\alpha v_{x}+\sigma s_{x}-\gamma u_{x}-\beta q_{y}+\beta q^{2} \\
& +(u-r)(\gamma q+\delta r-\beta s-\alpha v-\sigma s+\gamma u)] \xi^{-1},
\end{aligned}
$$




$$
\begin{gathered}
d_{2}=-\delta \xi-\beta q_{x} \xi^{-1}, \\
f_{2}=-\gamma \xi-2 \sigma s+\left(-\sigma s_{x}+\gamma u_{x}-\sigma s_{y}+\gamma u_{y}+\sigma q s\right. \\
\left.-\sigma u s+\gamma u^{2}\right) \xi^{-1}, \\
b_{2}+f_{2}=(\alpha+\gamma) \xi+2 \gamma q-\sigma s+\gamma u+\gamma v+\left(-\gamma q_{y}\right. \\
-\delta r_{y}+\beta s_{y}+\alpha v_{y}-\gamma q u-\beta q s-\gamma q v-\delta r v \\
\left.+\beta v s+\alpha v^{2}\right) \xi^{-1}, \\
a_{2}=-\sigma q+\sigma\left(q^{2}-q_{y}\right) \xi^{-1}, \\
q_{x}=q(r-u), \\
\left(q^{2}-q_{y}\right)_{x}=q^{2}-q_{y} .
\end{gathered}
$$

According to the TAH scheme, we form a $(2+1)$-dimensional integrable hierarchy

$$
\left(\begin{array}{c}
q \\
r \\
s \\
u \\
v
\end{array}\right)_{t_{n}}=\left(\begin{array}{c}
\mathscr{R}\left(c_{n+1}\right) \\
\mathscr{R}\left(d_{n+1}-a_{n+1}\right) \\
-\mathscr{R}\left(b_{n+1}+f_{n+1}\right) \\
\mathscr{R}\left(a_{n+1}\right) \\
\mathscr{R}\left(f_{n+1}\right)
\end{array}\right) .
$$

When we set $u=v=0$, (110) reduces to the $(2+1)$-dimensional integrable hierarchy (78). Hence, (110) is an expanding integrable model of (78).

A simple reduction of (110), when $n=1$, has the form

$$
\begin{aligned}
q_{t}= & \mathscr{R}\left(c_{2}\right) \\
= & -\gamma q_{x}-\delta r_{x}+\beta s_{x}+\alpha v_{x}+\sigma s_{x}-\gamma u_{x}-\beta q_{y} \\
& +\beta q^{2}+(u-r)(\gamma q+\delta r-\beta s-\alpha v-\sigma s+\gamma u), \\
r_{t}= & -\beta q_{x}+\sigma\left(q_{y}-q^{2}\right), \\
s_{t}= & \gamma q_{y}+\delta r_{y}-\beta s_{y}-\alpha v_{y}+\gamma q u+\beta q s+\gamma q v+\delta r v \\
& -\beta v s-\alpha v^{2}, \\
u_{t}= & \sigma\left(q^{2}-q_{y}\right), \\
v_{t}= & -\sigma s_{x}+\gamma u_{x}-\sigma s_{y}+\gamma u_{y}+\sigma q s-\sigma u s+\gamma u^{2},
\end{aligned}
$$

which has the constraint condition (109). If we further take $n=2,3, \ldots$, we obtain different $(2+1)$-dimensional integrable systems with their corresponding constraint conditions.

\section{Conclusions}

In the paper we have discussed two applications of the operator Lie algebra (15) and derived some new results. These include a second-order differential equation and its Lagrangians, the quasi-extremal equation and its general solutions, and the (1+1)-dimensional and (2+1)-dimensional integrable dynamical hierarchies as well as their expanding integrable models. We have adopted the vector Lie algebra $R^{7}$ to deduce the expanding integrable models of the integrable hierarchies, so that we can generate Hamiltonian structures of the expanding integrable models by the quadratic-form identity or the variational identity in $[14,15]$. It is worth further studying discretizations of the differential equations obtained in this paper. By applying some schemes in [16], we can further investigate the discretizations' exact solutions, symmetries, infinitely many conservation laws, and quasiperiodic solutions by following the ways in [17-32].

\section{Competing Interests}

The authors declare that they have no competing interests.

\section{Acknowledgments}

This work was supported by the National Natural Science Foundation of China (Grant no. 11371361), the Innovation Team of Jiangsu Province hosted by China University of Mining and Technology (2014) and the Fundamental Research Funds for the Central Universities (2013XK03), the Natural Science Foundation of Shandong Province (Grant no. ZR2013AL016), Hong Kong Research Grant Council (Grant no. HKBU202512), and Key Discipline Construction by China University of Mining and Technology (Grant no. XZD201602).

\section{References}

[1] D. Levi and P. Winternitz, "Symmetries and conditional symmetries of differential-difference equations," Journal of Mathematical Physics, vol. 34, no. 8, pp. 3713-3730, 1993.

[2] D. Levi and P. Winternitz, "Symmetries of discrete dynamical systems," Journal of Mathematical Physics, vol. 37, no. 11, pp. 5551-5576, 1996

[3] M. I. Bakirova, V. A. Dorodnitsyn, and R. V. Kozlov, "Symmetry-preserving difference schemes for some heat transfer equations," Journal of Physics A: Mathematical and General, vol. 30, no. 23, pp. 8139-8155, 1997.

[4] V. Dorodnitsyn, R. Kozlov, and P. Winternitz, "Continuous symmetries of Lagrangians and exact solutions of discrete equations," Journal of Mathematical Physics, vol. 45, no. 1, pp. 336359, 2004.

[5] V. Dorodnitsyn, "Noether-type theorems for difference equations," Applied Numerical Mathematics, vol. 39, no. 3-4, pp. 307$321,2001$.

[6] G. W. Bluman and S. Kumei, Symmetries and Differential Equations, vol. 81 of Applied Mathematical Sciences, Springer, New York, NY, USA, 1989.

[7] Y.-F. Zhang, L.-X. Wu, and W.-J. Rui, "A corresponding Lie algebra of a reductive homogeneous group and its applications," Communications in Theoretical Physics, vol. 63, no. 5, pp. 535$548,2015$.

[8] G. Z. Tu, "The trace identity, a powerful tool for constructing the Hamiltonian structure of integrable systems," Journal of Mathematical Physics, vol. 30, no. 2, pp. 330-338, 1989. 
[9] G. Z. Tu, R. I. Andrushkiw, and X. C. Huang, "A trace identity and its application to integrable systems of $1+2$ dimensions," Journal of Mathematical Physics, vol. 32, no. 7, pp. 1900-1907, 1991.

[10] Y. Zhang and W. Rui, "On generating (2+1)-dimensional hierarchies of evolution equations," Communications in Nonlinear Science and Numerical Simulation, vol. 19, no. 10, pp. 3454-3461, 2014.

[11] Y. F. Zhang and W. J. Rui, "A few super-integrable hierarchies and some reductions, super-Hamiltonian structures," Reports on Mathematical Physics, vol. 75, no. 2, pp. 231-255, 2015.

[12] Y. F. Zhang, H. W. Tam, and J. Q. Mei, "Some 2+1 dimensional super-integrable systems," Zeitschrift für Naturforschung A, vol. 70, no. 10, pp. 791-796, 2015.

[13] Y. Zhang and L. Wu, "Two (2+1)-dimensional expanding dynamical systems associated to the mKP hierarchy," Applied Mathematics and Computation, vol. 268, pp. 561-574, 2015.

[14] F. K. Guo and Y. F. Zhang, "The quadratic-form identity for constructing the Hamiltonian structure of integrable systems," Journal of Physics A: Mathematical and General, vol. 38, no. 40, pp. 8537-8548, 2005.

[15] W.-X. Ma and M. Chen, "Hamiltonian and quasi-Hamiltonian structures associated with semi-direct sums of Lie algebras," Journal of Physics A: Mathematical and General, vol. 39, no. 34, pp. 10787-10801, 2006.

[16] S. Y. Lou, "A note on the new similarity reductions of the Boussinesq equation," Physics Letters A, vol. 151, no. 3-4, pp. 133$135,1990$.

[17] S. Y. Lou, "Generalized symmetries and $W_{\infty}$ algebras in threedimensional Toda field theory," Physical Review Letters, vol. 71, no. 25, pp. 4099-4102, 1993.

[18] S. Y. Lou, "Symmetries of the Kadomtsev-Petviashvili equation," Journal of Physics A: Mathematical and General, vol. 26, no. 17, pp. 4387-4394, 1993.

[19] C. Cao, X. Geng, and Y. Wu, "From the special 2+1 Toda lattice to the Kadomtsev-Petviashvili equation," Journal of Physics. A. Mathematical and General, vol. 32, no. 46, pp. 8059-8078, 1999.

[20] C.-W. Cao and X.-X. Xu, "A finite genus solution of the Veselov's discrete Neumann system," Communications in Theoretical Physics, vol. 58, no. 4, pp. 469-474, 2012.

[21] C. Cao and X. Yang, "A (2+1)-dimensional derivative Toda equation in the context of the Kaup-Newell spectral problem," Journal of Physics. A. Mathematical and Theoretical, vol. 41, no. 2, Article ID 025203, 19 pages, 2008.

[22] X. G. Geng, H. H. Dai, and J. Y. Zhu, "Decomposition of the discrete Ablowitz-Ladik hierarchy," Studies in Applied Mathematics, vol. 118, no. 3, pp. 281-312, 2007.

[23] X. G. Geng, H. H. Dai, and C. Cao, "Algebro-geometric constructions of the discrete Ablowitz-Ladik flows and applications," Journal of Mathematical Physics, vol. 44, no. 10, pp. 45734588, 2003.

[24] E. G. Fan and Z. H. Yang, "A lattice hierarchy with a free function and its reductions to the Ablowitz-Ladik and Volterra hierarchies," International Journal of Theoretical Physics, vol. 48, no. 1, pp. 1-9, 2009.

[25] Z.-N. Zhu and H.-W. Tam, "Nonisospectral negative Volterra flows and mixed Volterra flows: lax pairs, infinitely many conservation laws and integrable time discretization," Journal of Physics. A. Mathematical and General, vol. 37, no. 9, pp. 31753187, 2004.
[26] D. J. Zhang and D. Y. Chen, "Hamiltonian structure of discrete soliton systems," Journal of Physics A: Mathematical and General, vol. 35, no. 33, pp. 7225-7241, 2002.

[27] Y. B. Suris, "On some integrable systems related to the Toda lattice," Journal of Physics A: Mathematical and General, vol. 30, no. 6, pp. 2235-2249, 1997.

[28] W.-X. Ma and B. Fuchssteiner, "Algebraic structure of discrete zero curvature equations and master symmetries of discrete evolution equations," Journal of Mathematical Physics, vol. 40, no. 5, pp. 2400-2418, 1999.

[29] Z. J. Qiao, " $r$-matrix and algebraic-geometric solution for integrable symplectic map," Chinese Science Bulletin, vol. 44, no. 2, pp. 114-118, 1999.

[30] Z. Zhu, H. Huang, and W. Xue, "Lax representations and integrable time discretizations of the DDKdV, DDmKdV, and DDHOKdV," Physics Letters. A, vol. 252, no. 3-4, pp. 180-190, 1999.

[31] Z.-N. Zhu, H.-Q. Zhao, and F.-F. Zhang, "Explicit solutions to an integrable lattice," Studies in Applied Mathematics, vol. 125, no. 1, pp. 55-67, 2010.

[32] W.-X. Ma, "Conservation laws of discrete evolution equations by symmetries and adjoint symmetries," Symmetry, vol. 7, no. 2, pp. 714-725, 2015. 


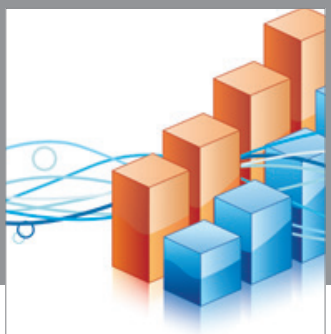

Advances in

Operations Research

vatem alat4

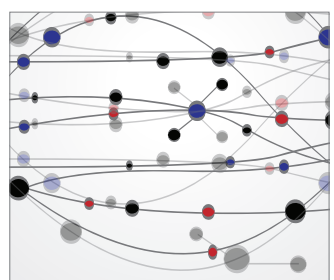

\section{The Scientific} World Journal
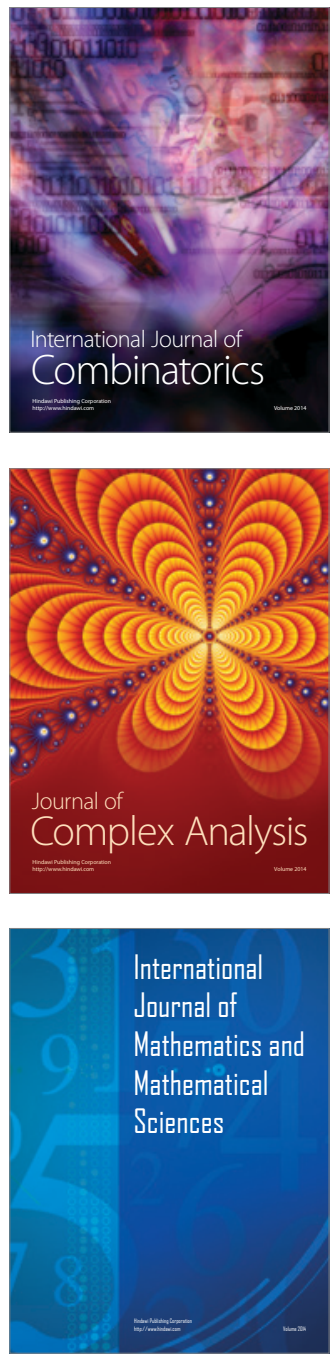
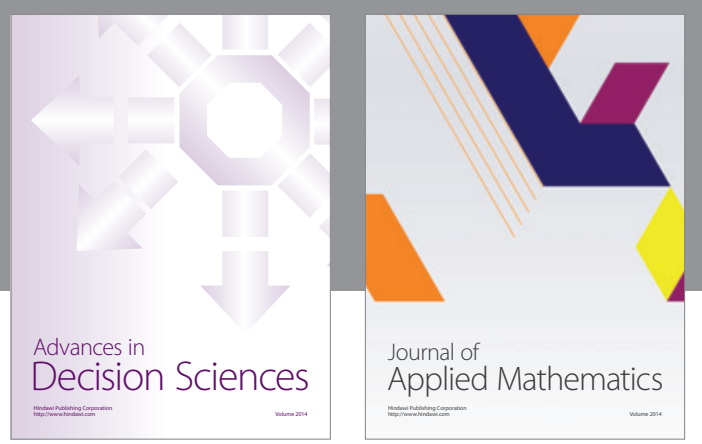

Algebra

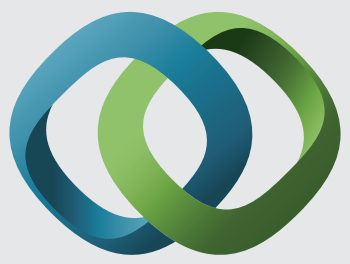

\section{Hindawi}

Submit your manuscripts at

http://www.hindawi.com
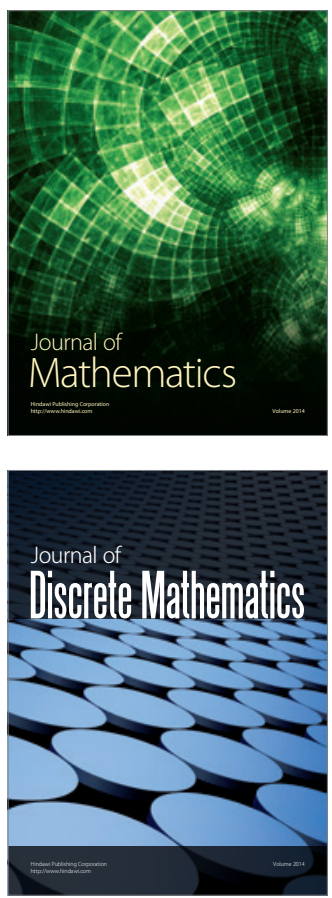

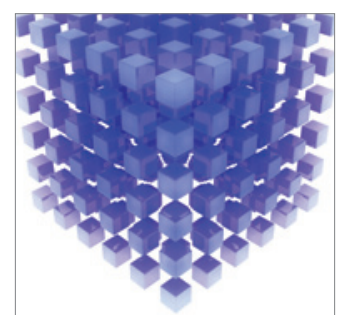

Mathematical Problems in Engineering
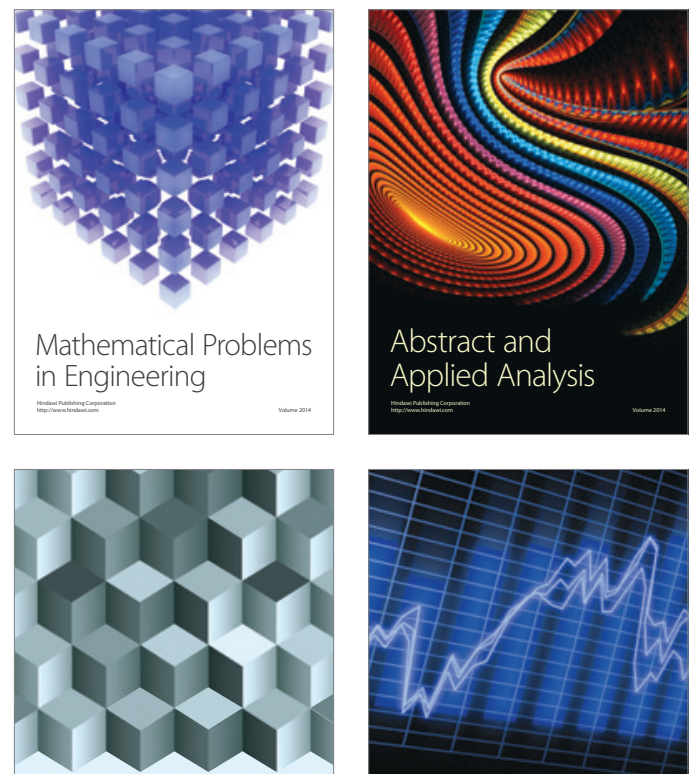

Journal of

Function Spaces

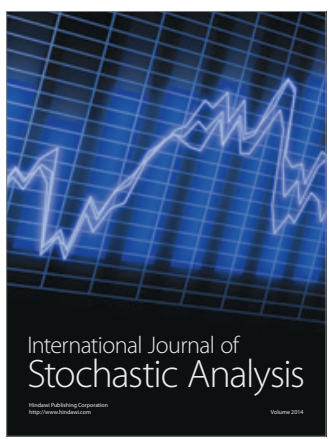

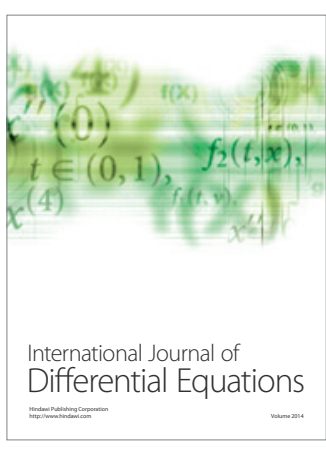
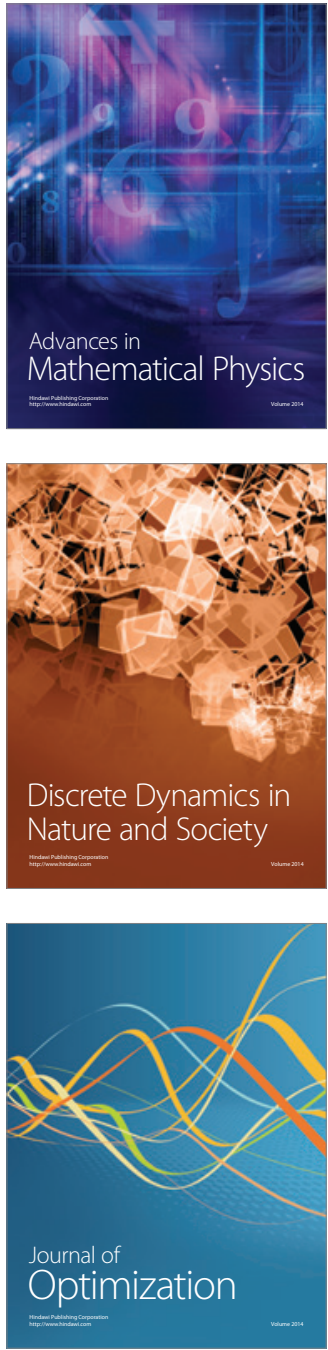